\title{
Los estudios sobre lectura y escritura en el contexto universitario. Una forma de abordar la realidad de la competencia lectora'
}

\author{
Reading and Writing Research in the University Context - \\ A way to Approach the Literacy Competence Reality
}

\author{
Ma ISABEL MORALES SÁNCHEZ \\ Universidad de Cádiz \\ España \\ isabel.msanchez@uca.es
}

La lectura y la escritura son dos actividades que marcan nuestra identidad y nuestro modo de aprehender el mundo que nos rodea, la forma de interpretarlo, de presentarlo, de transgredirlo, de reafirmarlo o, incluso, de negarlo. De hecho, podríamos decir que ambas son experiencias íntimas, pues requieren una previa confirmación de un yo que expresa, se emociona, empatiza y persuade. Puesto que ambas son inequívocamente sintomáticas de la personalidad y ayudan a configurarla -también de una actitud y aptitud ante el mundo- urge ser conscientes de la trascendencia que tiene nuestro posicionamiento como lectores o escritores sobre todo lo que circunda a nuestro alrededor, y, como consecuencia, atender y reconocer la importancia que tiene una formación de apoyo transversal e integrada, que permita un "entrenamiento" eficaz que complete nuestra experiencia en la construcción y comprensión de diversos tipos de discurso. La capacidad para interpretarlos conforma nuestro modo de responder a ellos y, por lo tanto, forma parte de los requisitos básicos que configuran nuestro pensamiento crítico. Discernir, interpretar, analizar y resolver son algunas de las operaciones que realizamos cuando leemos, pero ninguna de ellas se ejecuta definitivamente ni de forma absoluta, pues, la lectura es una experiencia dinámica, conectada a diferentes realidades, a contextos diversos, realizada por individuos dispares. También hemos de advertir de la existencia de niveles de dificultad y profundización, unidos a los constantes retos que las realidades textuales -incluidas las literarias- y los distintos procesos que las crean nos proponen. Ciertamente, un texto, sea de la índole que sea, encierra todo un universo de elementos lingǘsticos, semióticos y culturales que son articulados intencionadamente, al objeto de producir efectos y reacciones en el lector y, también, de persuadirlo, puesto que, cuando

\footnotetext{
${ }^{\text {I }}$ Para citar este artículo: Morales Sánchez, Ma Isabel (2020), Los estudios sobre lectura y escritura en el contexto universitario. Una forma de abordar la realidad de la competencia lectora. Allabe 2I . [www.revistaalabe.com] 
comunicamos, comunicamos también posicionamientos e intenciones. Un lector que no reacciona es un lector que no está leyendo o que no advierte aquello que lee, no descubre, no entiende las estrategias ni el escenario donde se sitúa al leer. Es por ello que trabajar en la comprensión crítica proporciona una dimensión de los discursos especialmente reveladora. Habitualmente hablamos del "descubrimiento" de la lectura, que no necesariamente se realiza en una etapa determinada ni exacta de nuestra vida, suponiendo un esfuerzo constante. A veces, este descubrimiento viene por casualidad, otras, por una necesidad concreta....pero cuando se hace, cuando lo comprendemos, vemos sobrevolar sobre el texto modos de pensar, de discutir, de argumentar, de razonar, esto es, todo lo que ayuda a conformar, desde el distanciamiento y el análisis, nuestro pensamiento. Por eso es imprescindible la formación lectora, por eso debe ser un eje esencial de cualquier nivel de formación, por eso, también, debe trabajarse con especial esmero en los niveles universitarios. El número que presentamos a continuación viene determinado por un interés común: profundizar en métodos, modos y formas de trabajar las competencias lectoescritoras desde diversas perspectivas y etapas de la formación, pero, en particular, abordar modos de enfrentar las necesidades reales que sobre competencia lectora detectamos, en el contexto universitario o inmediatamente anterior a él, espacios tan importantes para la capacitación de los futuros profesionales. Es preciso perfilar, en este sentido, acciones que atiendan al desafío de superar las barreras del conocimiento que han dividido al mismo, incluso, contraponiéndolo, en dos ámbitos familiarmente conocidos como "ciencias" y "letras", para enfocar el abordaje de la competencia lectoescritora como una capacidad transversal, entendida desde la recuperación del humanismo como un conjunto de saberes plurales desde los que construir mecanismos eficaces para trabajar y entrenar el espíritu crítico, con independencia de la profesión específica ejercida. En este sentido, consideramos imprescindible generar una reflexión que atienda a dos hechos esenciales: el conocimiento, desde todas sus perspectivas y enfoques, no sólo permite trabajar sobre el ciudadano, sino, también, sobre el profesional o los profesionales que van a formar a los futuros ciudadanos. Hablar de lectores en el espacio universitario no puede atender tan sólo a la vertiente personal, sino también a la responsabilidad profesional que más adelante deberán asumir. El segundo, que los profesionales no desarrollan su labor de forma aislada a la sociedad y, por lo tanto, tienen principios éticos que guían no sólo su condición como parte de ella, sino como agentes del conocimiento. A partir de este presupuesto, el abordaje de la competencia lectoescritora-sin necesidad de separar una de otra pues van íntimamente unidas-, en el contexto universitario, se ha convertido en prioridad urgente y necesita una reelaboración de sus presupuestos para convertirla en el catalizador de todas las destrezas, conocimientos y capacidades que permiten discernir identidades y posicionamientos, actitudes y razonamientos, a través de los distintos procesos que inundan nuestra realidad más cotidiana. Somos "identidad" cuando leemos y, también, cuando escribimos. Un ejemplo claro y siempre recurrente en los últimos años es la constante alusión a cómo las nuevas tecnologías están transformando nuestros hábitos lectores. Pero esta transformación es más profunda si cabe, pues están cambiando el 
modo en cómo nos relacionamos y actuamos socialmente. La transformación en la forma de comunicación es y está siendo trascendental, no siendo pocas las voces que reclaman una atención a la vertiente emocional y empática, al objeto de salvar hábitos incipientes originados tales como el aislamiento o la ocultación del yo. Atender y comprender cómo son nuestros espacios de sociabilidad, vuelvo a insistir, tanto personales como profesionales, resulta imprescindible, como resulta igual de obligatorio desarrollar estrategias personales que nos permitan desplegar las nuevas habilidades requeridas por el entorno. Las nuevas tecnologías se han convertido en el tópico que justifica, con razón, todo lo que en cuanto a nuestro alrededor ha sido mutado, para bien o para mal. El hecho es que el acceso múltiple y multiforme a la información, la existencia de plataformas de comunicación, la difusión de noticias en la red y un largo etc. de elementos ponen al sujeto en una confluencia de caminos llena de retos distintos, variables e inesperados. Todo ello ha generado lenguajes diversos, espacios controvertidos y un buen número de herramientas que gestionar y sobre las que decidir. Desde el punto de vista de la investigación, parece perfectamente lógico que se preste atención a los nuevos discursos y realidades, que impregnan todas las esferas prácticas y teóricas. Este nuevo marco ha cambiado, por ejemplo, el modo en cómo nos relacionamos con nuestras amistades; la forma de viajar, el diseño del viaje y la actitud del viajero; los modos de divulgar y enseñar la cultura y la lengua -también el propio sentido que le damos a ambas-; las formas literarias, el arte y sus espacios de representación y difusión, incluso, en la relación del creador con su espectador o lector.

Los estudios recogidos en este número abordan alguno de los espacios mencionados desde perspectivas diferentes, mostrándonos la lectura en toda su complejidad. Es cierto que sólo suponen una muestra, pero, creemos, suficientemente ilustrativa de la profundidad del iceberg. Las líneas delimitadas giran alrededor de la literatura, de los medios digitales, de la literacidad y de los retos de la comprensión lectora.

Felisa Ferraz y Kara Tober se acercan a la literatura como elemento complejo, capaz de permeabilizar los comportamientos culturales moduladores de nuestros nuevos modos de apropiación y observación, dando buena muestra de la disparidad con los que puede abordarse. En el primer caso, guían el recorrido, en sentido literal, las rutas pirenaicas literarias, un nuevo espacio patrimonial y de ocio que permite realizar de manera efectiva el trasvase entre los paisajes creados por la literatura y los lugares y espacios de la cultura arraigada a la tradición, las costumbres y a la tierra. Este lector que reconoce lo leído en la realidad y que lo transforma, puede ser dirigido, en dirección contrapuesta, hacia el desconcierto. Es así como Kara Tober nos acerca a la novela de Santiago Roncagliolo Tan cerca de la vida (2OII), una exploración sobre la denominada "narración perturbadora", un modo discursivo percibido como absolutamente intencionado, marcado por la influencia del contexto transmedial, en el que las distintas estrategias pretenden imbuir al lector, zarandeándolo, en una a una historia compleja, cuyos efectos van de la empatía a la desorientación, de la realidad a la ciencia ficción. Por su parte, Cristina Rentería nos introduce en la obra de Pablo Gutiérrez Democracia (20I2) para explicar lo que 
viene a denominar Españistown, un constructo social propio de la sociedad española de la transición, vinculado a un modo de vida y un imaginario que ayuda a entender la realidad económica, política y social de nuestro país hasta la crisis de 2008. Estos tres acercamientos a la crítica literaria nos ayudan a comprender no sólo obras y autores determinados, sino cómo la lectura no puede abstraerse de todos los discursos que se entrecruzan en ellas desde el posicionamiento del autor, desde el texto y desde la mirada del lector.

Desde aquí y centrados en la línea de estudio de la lectura como actividad social y cultural, el número aborda dos perspectivas esenciales: por una parte, la lectura desde el punto de vista jurídico e institucional como derecho y práctica educativa, tal y como ofrece el estudio de Aldo Ocampo, centrado en los años I97O-I985, a través del análisis de las políticas sobre el libro y desde el concepto de educación inclusiva, identificando las principales condiciones que hacen de la lectura un instrumento esencial de equilibrio ante las distintas formas de desigualdad. Por otra, común a la mayor parte de los estudios presentados, la transformación de los hábitos lectoescritores en primaria, secundaria y universidad, por la irrupción de las prácticas digitales y los entornos tecnológicos actuales. Esta última línea viene encabezada por el estudio de casos realizado por Domingo Albarracín sobre hábitos ortográficos en primaria e influencia mediática en escritura creativa. El estudio resulta especialmente interesante por ser extrapolable a tres espacios: la oralidad, la escritura y la lectura, ofreciendo resultados contundentes y precisos en el estudio de la producción discursiva y conceptual. A continuación, Marcela Cabrera y Sergio Caruman exploran el contexto de secundaria, atendiendo con interés aquellos aspectos que trascienden del mismo de cara a la identificación de los niveles de competencia lectora en fases preuniversitarias. Su investigación, dirigida por el interés de medir la eficacia concreta de recursos, analiza los resultados de la denominada Prueba de Selección Universitaria-Lenguaje, usada en Chile para la selección de estudiantes y cuyas conclusiones revelan, entre otros aspectos, dificultades en la comprensión lectora a la hora de identificar perspectivas, vislumbrar la estructura del texto o descubrir conceptos inmersos en él. Por último, el siguiente grupo de artículos, centrados en el ámbito universitario, responde a varias vertientes y orientaciones de estudio, abarcando desde prácticas concretas en el aula con dinámicas digitales, hasta el diagnóstico de las iniciativas de promoción lectora articuladas en este ámbito, sin olvidar, los retos actuales que las tecnologías plantean a los tradicionales clubes de lectura. Yolanda de Gregorio y Mario de la Torre, han integrado en sus experiencias docentes espacios digitales, en concreto, la literatura digital -en el primer caso- y el fenómeno booktube, en el segundo. Estas interesantes prácticas dan cuenta tanto de las ventajas como de las dificultades de la utilización de estos elementos en el aula, por cuanto, paradójicamente, resultan extraños a los alumnos detectándose, incluso, reticencias a su exploración.

Juan Pedro Martín Villarreal nos ofrece una propuesta tan compleja y arriesgada como necesaria, al enfrentar el diagnóstico de las actividades de lectura realizada, esta vez en la Universidad de Cádiz. Este tipo de estudios resultan absolutamente necesarios por cuanto permiten conciliar lo que las iniciativas individuales con las institucionales, dando 
una imagen certera de cuánto se hace o no realmente por la proporción de la lectura y el abordaje de la competencia lectora en ámbitos, grados y espacios diferentes. De hecho, algo similar ocurre con la implantación de los clubes de lectura universitarios, una actividad en declive en los últimos años y que requiere de una dirección y organización eficaz por parte de la institución y de los profesionales que la conformamos, tanto en el ámbito de la docencia como en el de la biblioteca. Lo sugerente de una propuesta como esta viene de la mano de la primera cuestión que plantean María Antonia Moreno, Araceli García y Raquel Gómez: si la biblioteca pública en España ha apostado en los últimos años por los clubs de lectura, tanto en su modalidad presencial como virtual o en la nube, demostrando su eficacia... ¿debería la universidad retomar esta tendencia y adaptar las buenas prácticas al ámbito universitario?

Para finalizar, cierra el apartado de ensayos el acercamiento de Alicia Mariscal al contexto de la enseñanza de las segundas lenguas en la universidad, para mostrarnos una experiencia de fomento de la lectoescritura en el ámbito de la lingüística aplicada. Con una metodología basada en la lectura de textos tanto en lengua materna como en la extranjera, esta propuesta pretende fomentar la competencia en la escritura y traducción de textos académicos propios del contexto universitario.

Completan el número los habituales apartados Textos y Documentos y Álabes imprescindibles, el primero, con el texto de Luca Toschi, dedicado a la experiencia -su experiencia- lectora, una enriquecedora reflexión construida alrededor del recuerdo de la puerta de la biblioteca paterna, toda una metáfora del descubrimiento, de la trasgresión y de la curiosidad. Una iniciación a la lectura que abre fronteras, mentes, pensamientos, muros infranqueables, que revela y humaniza a través de la palabra, revelada como infinita. En segundo lugar, Álabes imprescindibles, está dedicado a la poeta, recién fallecida, Mariluz Escribano, gran escritora de la memoria y una de las figuras esenciales de la poesía española de los últimos cuarenta años. Escribano, docente de Didáctica de la Lengua y la Literatura durante cuarenta años, y una de las fundadoras del área, era muy consciente de la importancia de trabajar con entusiasmo la educación literaria para formar maestros comprometidos. Es un acto de justicia a una persona de referencia en el área y también una invitación a la relectura de su obra en cualquiera de sus vertientes -poética o narrativa-, tan evocadora y conciliadoras ambas en igual medida.

Número completo, por lo tanto, en cuanto a sugerencias y líneas, que permite pensar y repensar la lectura, sus retos, su problemática, así como algunas de las soluciones que tenemos a nuestro alcance.

Mª Isabel Morales Sánchez

Universidad de Cádiz 